\title{
Suppression of DNA-PKes and Ku80 individually and in combination: Different effects of radiobiology in HeLa cells
}

\author{
LIANG ZHUANG ${ }^{1}$, YANG CAO ${ }^{2}$, HUIHUA XIONG $^{1}$, QINGLEI GAO $^{2}$, ZHE CAO $^{1}$, \\ FEI LIU ${ }^{1}$, HONG QIU ${ }^{1}$, SHIYING YU ${ }^{1}$ and XIAOYUAN HUANG ${ }^{2}$ \\ ${ }^{1}$ Cancer Center, ${ }^{2}$ Cancer Biology Research Center, Tongji Hospital, Tongji Medical College, \\ Huazhong University of Science and Technology, Wuhan Hubei 430030, P.R. China
}

Received February 1, 2011; Accepted April 15, 2011

DOI: 10.3892/ijo.2011.1041

\begin{abstract}
DNA-dependent protein kinase (DNA-PK), including $\mathrm{Ku} 80, \mathrm{Ku} 70$ and DNA-PK catalytic subunit (DNA-PKcs), is the key protein in non-homologous end-joining (NHEJ) after DNA double-strand breaks (DSBs) appear. In this study, small hairpin interfering RNAs (siRNAs) targeting Ku80 and DNAPKcs were used both individually and in combination, to explore the effects of these DSB proteins on HeLa cell functional changes after X-ray irradiation. HeLa cells co-transfected with Ku80-siRNA and DNA-PKcs-siRNA were more radiosensitive than the ones transfected individually. HeLa in the absence of Ku80 and pretreated with LY294002, a chemically specific PI 3-kinase inhibitor, resulted in cells that were even more sensitive to X-rays than HeLa/Ku80-siRNA transfected with DNAPKcs-siRNA. The cells inhibited by Ku80 either individually or in combination with DNA-PKcs showed cell accumulation in the $\mathrm{G} 2 / \mathrm{M}$ phase $48 \mathrm{~h}$ post-irradiation, similarly to control cells. However, cells transfected with DNA-PKcs-siRNA or pretreated with LY294002 had a prolonged G2/M delay, suggesting the accumulation of significant un-repaired DNA damage following inhibition of DSB repair proteins. In conclusion, these data indicate that the role of Ku80 in DSB repair could be compensated by other DSB repair proteins; co-inhibition would be a suitable strategy to enhance the radiosensitivity of cancer cells.
\end{abstract}

\section{Introduction}

DNA double-strand breaks (DSBs) are generally regarded as the most lethal of all DNA lesions after radiation and are repaired by two major repair pathways: homologous recombination (HR) and non-homologous end-joining (NHEJ) (1-3). It is generally

Correspondence to: Dr Xiaoyuan Huang, Cancer Biology Research Center, Tongji Hospital, Tongji Medical College, Huazhong University of Science and Technology, Wuhan 430030, P.R. China

E-mail: huangxy@tjh.tjmu.edu.cn

Key words: Ku80, DNA-PK, LY294002, radiosensitivity, cell cycle believed that NHEJ plays a more important role than HR in mitotically replicating cells, especially in human cells (4). The known NHEJ proteins include DNA-dependent protein kinase (DNA-PK), ligase IV, and its cofactor XRCC4, among others. DNA-PK is a serine threonine kinase that contains a $\mathrm{Ku}$ heterodimer (Ku70 and Ku80, Ku80 also named Ku86) and a DNA-PK catalytic subunit (DNA-PKcs), and is crucial to the NHEJ process and in the maintenance of telomere stability $(3,5)$. Correspondingly, the ataxia-telangiectasia mutated (ATM) protein plays a main role in HR that is only a pre-emergency DSB repair mechanism in human cells during late $S, G 2$ and $M$ phase $(6,7)$. In contrast, NHEJ is present at all times during the entire cell circle, especially in the G0/G1 and early S stages $(8,9)$. When DSBs are produced by irradiation, the Ku heterodimer, which has a high affinity for DNA, can bind preferentially to the free DNA ends, possibly to prevent nuclease attack; subsequently, the conformation of $\mathrm{Ku}$ changes, allowing it to interact with the DNA-PKcs/Artemis complex (5). This complex acts as an endonuclease at both the $5^{\prime}$ and $3^{\prime}$ overhangs, and trims the $5^{\prime}$ overhangs, resulting in a blunt end. In contrast, the $3^{\prime}$ overhangs are clipped with a preference for leaving a 4- or 5-nuleotide single-stranded overhang (10). After autophosphorylation, DNA-PKcs recruits XRCC4/ligase IV complex to process the DNA ends and to initiate re-ligation to form a single DNA molecule $(5,11,12)$. Thus, targeted inhibition of DSB repair proteins is an attractive approach in the development of potent radiation therapy strategies $(13,14)$.

Previous studies have investigated the consequences of the deficiencies of individual components of DNA-PK complex for DSB repair. However, only a few investigations have focused on co-inhibition. For prokaryotic or lower eukaryotic cells, the ability to obtain and study double or even triple mutants has been useful, but such mutants are not easily obtained for mammalian cells, as sometimes even single (let alone multiple) mutant phenotypes prove lethal (15). RNA interference (RNAi), which can highly specifically target gene silencing in mammalian cells using small interfering RNA (siRNA), has become a powerful tool in studying the cell function of any gene $(16,17)$. In this study, siRNA targeting Ku80 and DNA-PKcs were used both individually and in combination, to explore the effects of these DSB proteins on HeLa cell functional changes after X-ray irradiation. Because DNA-PKcs and ATM are both members of the PI 3-kinase gene family (18), a chemically 
specific PI 3-kinase inhibitor named as LY294002, which can increase radiosensitivity by blocking the activity of both DNA-PKcs and ATM $(19,20)$, was applied in our study.

\section{Materials and methods}

Cell line and cell culture. Human cervical adenocarcinoma cell line HeLa was obtained from the China Center for Type Culture Collection (CCTCC, Wuhan, China). The cells were grown in Dulbecco's modified Eagle's medium (Invitrogen, USA) supplemented with $10 \%$ fetal bovine serum (Invitrogen), $50 \mathrm{U} / \mathrm{ml}$ penicillin, and $50 \mu \mathrm{g} / \mathrm{ml}$ streptomycin. The HeLa cells were maintained in a humidified $37^{\circ} \mathrm{C}$ incubator with $5 \%$ $\mathrm{CO}_{2}$, fed every two to three days with a complete medium (contain 10\% FBS).

Plasmid construction. The pGenesil-1 (which was pEGFP-C1 inserted into a human U6 promoter by Genesil Biotechnology Co., Ltd, Wuhan, China) was used for the construction. The fragments, which transcribed the siRNA, are shown below (nucleotides and the sense and antisense sequences that will form a hairpin structure upon transcription inside cells are underlined). They were designed using a computer program available on the Internet (http://www.ambion.com/ techlib/misc/siRNA_finder.html) and the principle reported by (17) Ku80-siRNA was the sense: 5'-GATCCGACAGA CACCCTTGAAGACTTCAAGACGGTCTTCAAGGGTGT CTGTCTTTTTGTCGACA-3' and the antisense: 5'-AGCTT GTCGACAAAAAGACAGACACCCTTGAAGACCGTCTT GAAGTCTTCAAGGGTGTCTGTCG-3'. Negative-siRNA was the sense: 5'-GATCCGACTTCATAAGGCGCATGCTT CAAGACGGCATGCGCCTTATGAAGTCTTTTTGTCG ACA-3' and the antisense: 5'-AGCTTGTCGACAAAAAGACT TCATAAGGCGCATGCCGTCTTGAAGCATGCGCCTTA TGAAGTCG-3'. These above siRNA-encoding complementary single-stranded oligonucleotides, which were hybridized to give BamHI- and HindIII-compatible overhangs, then were ligated into pGenesil-1. Bacterial colonies were pooled and used for the plasmid preparation. The positive clones were confirmed by sequencing. The constructed plasmid, which transcripted DNA-PKcs-siRNA, was presented by Dr Xiao-Yu Tian, who applied RNAi-Ready pSIREN-DNR-DsRedExpress Donor Vector (Clontech, USA), which could express red fluorescent proteins for construction according to the manufacturer's protocol. The siRNA-encoding complementary single-stranded oligonucleotide fragment, which had BamHI and EcoRI overhang, had sense: 5'-GATCCCTTTATGGTGG CCATGGAGTTCAAGAGACTCCATGGCCACCATAAAG TTTTTTGGAAG-3' and antisense: 5'-AATTCTTCCAAAA AACTTTATGGTGGCCATGGAGTCTCTTGAACTCCAT GGCCACCATAAAGG-3'.

Transfection of cells. Plasmids were transfected into cells according to the manufacturer's protocol of Lipofectamine ${ }^{\mathrm{TM}}$ 2000 (Invitrogen). In short, one day prior to transfection, cells were seeded without antibiotics in a 6 -well plate at $3 \times 10^{5}$ cells/ well, corresponding to a density of $70-80 \%$ at the time of transfection. Plasmids (3 $\mu \mathrm{g})$ and $6 \mu \mathrm{l}$ Lipofectamine 2000 were mixed with DMEM without FBS and incubated for $5 \mathrm{~min}$ at room temperature. These reagents were combined and incubated for 20 min before being added to the cells in DMEM without FBS, penicillin or streptomycin. Cells were incubated at $37^{\circ} \mathrm{C}$ for $4 \mathrm{~h}$, then fresh DMEM with $10 \%$ FBS was added. While the cells that transfected Ku80-siRNA and negative-siRNA were planted into the 6-well plates again at $1 \times 10^{5}$ cells/well, DMEM with $10 \%$ FBS and $800 \mu \mathrm{g} / \mathrm{ml} \mathrm{G418}$ (Gibco, USA) were added, resulting in a concentration of G418 that decreased to $400 \mu \mathrm{g} / \mathrm{ml}$ when the cell clones were formed after 8-12 days of growth. The clones expressing GFP were selected out to maintain cultivation. Finally, two stable transfected HeLa cell clones (named HeLa/Ku80-siRNA and HeLa/ Neg-siRNA) were collected for the next study. In order to ascertain the transfection efficiencies of cells stably transfected with siRNA-encoding plasmids, the green fluorescence of these cells was quantified by fluorescence-activated cell sorting (FACS) analysis. Cells (including $\mathrm{HeLa} / \mathrm{Neg}$-siRNA and $\mathrm{HeLa} / \mathrm{Ku} 80$-siRNA) transfected with DNA-PKcs-siRNA were harvested at 24,48 and $72 \mathrm{~h}$ after transfection for Western blot analysis.

Western blot analysis. Cells were harvested after transfection and whole cell proteins were isolated via M-PER ${ }^{\mathrm{TM}}$ mammalian protein extraction reagent (Pierce, Rockford, IL). Protein concentrations were determined with a BCA protein assay kit (Pierce) according to the manufacturer's instructions. An equal amount of total protein $(50 \mu \mathrm{g})$ from each lysate was loaded onto 6-10\% SDS-polyacrylamide gel. After electrophoresis, the separated proteins were transferred to nitrocellulose membranes, which were then blocked with TBS-T $(0.1 \mu \mathrm{mol} / 1$ Tris- $\mathrm{HCl}, \mathrm{pH} 7.5 ; 0.9 \%$ sodium chloride; $0.05 \%$ Tween 20 ) containing $5 \%$ powdered non-fat milk for $1 \mathrm{~h}$ at $37^{\circ} \mathrm{C}$. Then this was incubated with mouse anti-human monoclonal antibodies against Ku80 (NeoMarkers, 1:2,000 dilution), DNA-PKcs (NeoMarkers, 1:2,000 dilution), or $\beta$-actin (Santa Cruz, 1:2,000 dilution) at $4^{\circ} \mathrm{C}$ overnight. After washing with TBS-T for $4 \times 15 \mathrm{~min}$, nitrocellulose membranes were incubated with 1:5,000 dilution horseradish peroxidase (HRP)-labeled goat anti-mouse second antibody for $1 \mathrm{~h}$ at room temperature and washed with TBS-T for $4 \times 15$ min. Immunodetection was performed using SuperSignal West Femto Maximum Sensitivity Substrate (Pierce) and the density of the bands in the resulting film was quantified using the NIH image analysis.

Clonogenic survival assay. Cells were plated in triplicate onto $60-\mathrm{mm}$ dishes at the required concentration to get 50-100 colonies per dish and were allowed to attach for $24 \mathrm{~h}$. $\mathrm{HeLa}, \mathrm{HeLa} / \mathrm{Neg}$-siRNA, HeLa/Ku80-shNRA cells and cells pretreated with $50 \mu \mathrm{mol} / 1 \mathrm{LY} 294002$ (Promega) for $30 \mathrm{~min}$ or transient were transfected with DNA-PKcs-siRNA for $48 \mathrm{~h}$ and were exposed to $0,1,2,3,4,6$, and 8 Gy X-ray. Then the cells were cultured for 10 to 14 days in $5 \% \mathrm{CO}_{2}$ to get viable colonies. The colonies were stained with $0.5 \mathrm{ml}$ of $0.01 \%$ crystal violet (Sigma-Aldridge) solution for $1 \mathrm{~h}$ and were counted by using a microscope (x40 magnification). A viable colony was defined as having at least 50 cells after 10 days of growth. Colonies were counted from each triplicate sample and presented as average values $\pm \mathrm{SD}$. The surviving fraction of the treated cells was normalized to the plating efficiency of control (no irradiation) cells. Cell survival was plotted as a function of dose and fitted using the linear quadratic model $S F=\exp \left(-\alpha D-\beta D^{2}\right)$, 

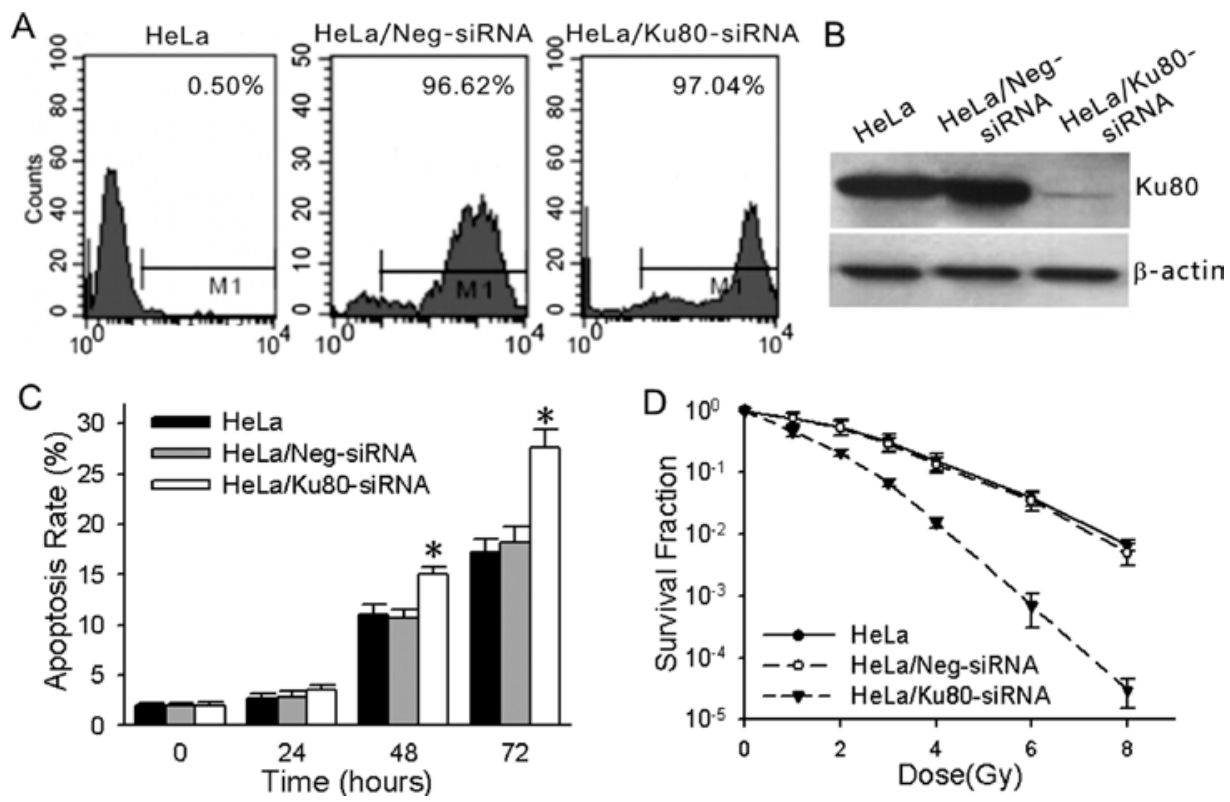

Figure 1. Effects of Ku80-siRNA on radiosensitivity of HeLa cells. (A) Transfection efficiencies of HeLa cells stably transfected with Ku80-siRNA and negative-siRNA-encoding plasmids that constitutively expressed green fluorescent protein (GFP). Cell clones stably transfected with siRNA-encoding plasmids were collected and green fluorescence was quantified by FACS analysis. (B) Western blot analysis of the Ku80 protein expression in HeLa cells transfected with Ku80-siRNA and Neg-siRNA. (C) HeLa cells were transfected with Ku80-siRNA and Neg-siRNA was treated with 6 Gy X-ray for 24, 48 and 72 h. The apoptosis rate was analyzed by FACS, "P<0.05. (D) HeLa cells were transfected with Ku80-siRNA and Neg-siRNA was exposed to 0 to 8 Gy X-ray prior to assessment of colony formation. The clonogenic survival assays were performed in triplicate. Standard errors are shown by error bars.

where $\mathrm{SF}$ is the cell survival, $\mathrm{D}$ is the dose of radiation, and $\alpha$ and $\beta$ are constants. SF2 (surviving fraction of cells at $2 \mathrm{~Gy}$ ) was calculated from the actual data. Dose-reduction factors (DRFs), the factor by which the dose of the radiation can be reduced in the presence of the sensitizing agent to achieve the same level of cell killing in the absence of the sensitizing agent, were calculated as the dose required to achieve $10 \%$ cell survival (90\% clonogenic cell killing) from the fitted clonogenic survival curves for the transfected cells.

Irradiation and cell cycle analysis. Cells were plated and pretreated with LY294002 at $50 \mu \mathrm{mol} / 1$ for $30 \mathrm{~min}$, and then were transported in a $37^{\circ} \mathrm{C}$ insulated container in sealed flasks and exposed to 6 Gy $6 \mathrm{MV}$ X-ray (300 cGy/min). The cells were stably transfected with siRNA-encoding plasmids or transient transfected with DNA-PKcs-siRNA-encoding plasmid for $48 \mathrm{~h}$. For the determination of cell cycle distribution, the cells were harvested after irradiation for $4 \mathrm{~h}$ (not for cells pretreated with LY294002), 24, 48 and $72 \mathrm{~h}$, washed with PBS, and fixed in $75 \%$ ice-cold ethanol overnight at $-20^{\circ} \mathrm{C}$. After being washed in PBS, the cells were treated with $50 \mu \mathrm{g} / \mathrm{ml}$ propidium iodide (PI) and $1 \mathrm{mg} / \mathrm{ml} \mathrm{RNase} \mathrm{A}$ for $30 \mathrm{~min}$ at $37^{\circ} \mathrm{C}$. Subsequent analyses of the apoptosis rate (Sub G1) and cell cycle distribution were performed using CellQuest software (BD Bioscences, Heidelberg, Germany).

Statistical analysis. SPSS v11.5 software was used for all statistical procedures. Data were presented as means \pm SD of at least triplicate experiments and a two-tailed unpaired t-test was used to compare the statistical significance of the differences in the data between the two groups. Differences at $\mathrm{P}<0.05$ were considered significant.

\section{Results}

Effects of Ku80-siRNA on radiosensitivity of HeLa cells. The plasmids that constitutively expressed green fluorescent protein (GFP) and could transcript Ku80-siRNA and negativesiRNA, were stably transfected into HeLa cells, named HeLa/ Ku80-siRNA and HeLa/Neg-siRNA, respectively. The transfection efficiencies of HeLa/Ku80-siRNA and HeLa/ Neg-siRNA were detected by FACS analysis and were all above $95 \%$, with the efficiency of $\mathrm{HeLa} / \mathrm{Ku} 80$-siRNA reaching $97.04 \%$ (Fig. 1A). More than $96 \%$ of the Ku80 protein was knocked down by Ku80-siRNA, while the Ku80 protein of $\mathrm{HeLa} / \mathrm{Neg}$-siRNA failed to decrease (Fig. 1B). These data indicate that the siRNA targeting of Ku80 was specific and the plasmid vector had no effect on Ku80 protein expression. $\mathrm{HeLa} / \mathrm{Neg}$-siRNA was used as control in the next study. HeLa cells were transfected with Ku80-siRNA and Neg-siRNA was treated with 6 Gy X-ray for 24, 48 and $72 \mathrm{~h}$. The apoptosis rate of $\mathrm{HeLa} / \mathrm{Ku} 80$-siRNA was significantly higher than that of HeLa and HeLa/Neg-siRNA ( $\mathrm{P}<0.05)$ (Fig. 1C). A clonogenic survival assay showed that HeLa cell silencing of Ku80 was more sensitive to X-rays than were the control cells (Fig. 1D).

Knockdown of DNA-PKcs by siRNAs after Ku80 silencing. Reconstructed DNA-PKcs-siRNA-transcripted plasmid, which could express red fluorescence protein in the host cell, was transfected into HeLa/Neg-siRNA and $\mathrm{HeLa} / \mathrm{Ku} 80$ siRNA cells (Fig. 2A). Protein extracts were obtained at 24, 48 and $72 \mathrm{~h}$ after transfection and a Western blot analysis was performed for the DNA-PKcs protein and normalized to $\beta$-actin expression. The results disclosed that $48 \mathrm{~h}$ post-transfection was the time point at which the greatest DNA-PKcs protein 
A

A $\mathrm{HeLa} / \mathrm{Ku} 80-$ SiRNA

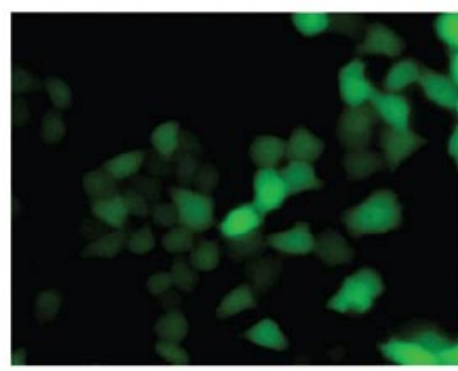

HeLa/DNA-PKcs-siRNA

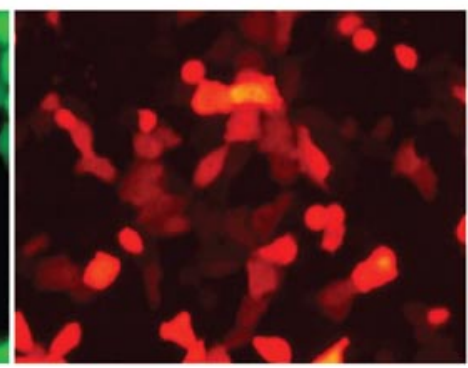

HeLa/Ku80-siRNA

DNA-PKCs-siRNA

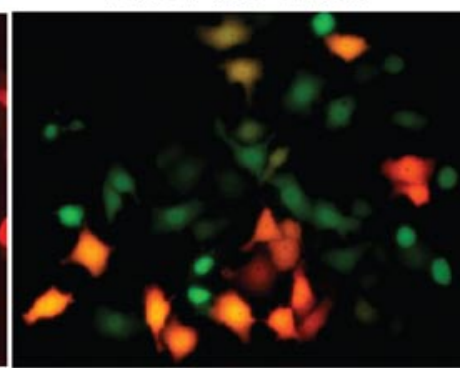

B

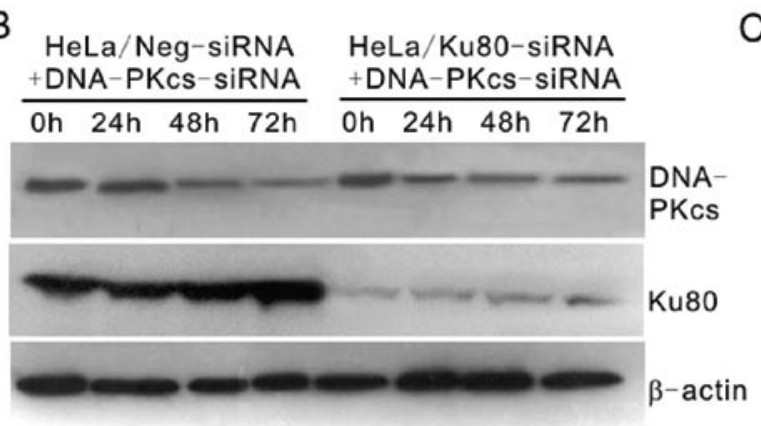

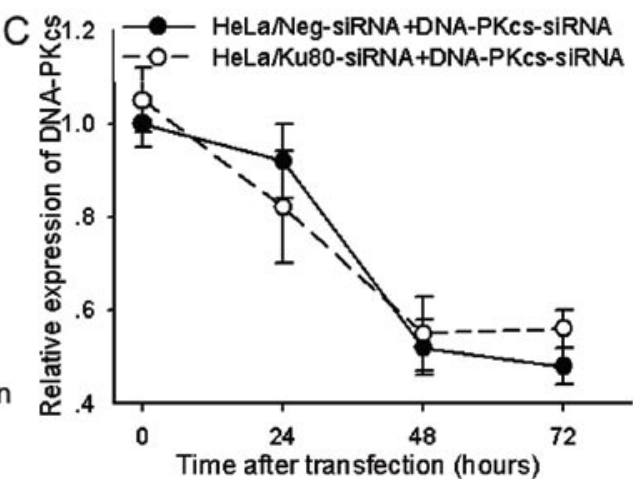

Figure 2. DNA-PKcs was down-regulated by DNA-PKcs targeting siRNA. (A) Reconstructed DNA-PKcs-siRNA-transcripted plasmid that expressed red fluorescence protein was transfected into $\mathrm{HeLa} / \mathrm{Ku} 80$-siRNA cells (x200). (B) Cell extracts were obtained from 24 to $72 \mathrm{~h}$ post-transfection and target proteins were detected by Western blot analysis and normalized to $\beta$-actin expression. (C) The density of bands was quantified by NIH image analysis and the data presented as mean values of each data point \pm standard error $(n=3)$.

down-regulation occurred (about 50\% lower) (Fig. 2B and C). No obvious changes in Ku80 protein were found at any time, either for the HeLa/Neg-siRNA or HeLa/Ku80-siRNA cells. Thus, the DNA-PKcs-targeting siRNA did not affect the expression of Ku80 protein and also suggested that Ku80 protein did not vary in $\mathrm{HeLa} / \mathrm{Ku} 80$-siRNA after a long-time culture after the cell line was established. In the next experiment, cells transfected with DNA-PKcs-siRNA for $48 \mathrm{~h}$ were analyzed by clonogenic survival assay and apoptosis rate and cell cycle analysis after X-ray irradiation.

HeLa cells cotransfected with Ku80-siRNA and DNA-PKcssiRNA in combination were more radiosensitive than either individually. The apoptosis analysis displayed that the apoptosis rate of HeLa cells cotransfected with Ku80-siRNA and DNA-PKcs-siRNA were higher than that of other cells $(\mathrm{P}<0.05$, Fig. 3A). The clonogenic assay showed that HeLa and HeLa/ Neg-siRNA had similar results in terms of SF2 and $\alpha$ values (Fig. 3B, Table I), confirming the notion that the plasmid vector had no effect on radiosensitivity either. In order to remove the effect of the plasmid vector, HeLa/Neg-siRNA was regarded as the control cells. As shown above, HeLa/Ku80-siRNA had 96\% Ku80 protein knockdown, meaning that the function of NHEJ would be nearly completely inhibited in normal inference. But when HeLa/Ku80-siRNA cells were transfected with DNAPKcs-siRNA, its SF2 value became significantly decreased (Fig. 3B, Table I) at only $0.08 \pm 0.01$, which is far lower than $\mathrm{HeLa} / \mathrm{Ku} 80$-siRNA $(0.20 \pm 0.05)$ and HeLa transfected only with DNA-PKcs-siRNA (0.25 \pm 0.03$)$. The DRF of $10 \%$ cell survival compared to HeLa/Neg-siRNA even reached 2.47. The $\alpha$ values of these cell lines had opposite change patterns (as shown in Table I), again showing that HeLa cells co-transfected with Ku80-siRNA and DNA-PKcs-siRNA were more radiosensitive than the ones transfected individually.

The cell cycle distributions of HeLa cells cotransfected with Ku80-siRNA and DNA-PKcs-siRNA showed interesting changes after 6 Gy X-ray irradiation. Because HeLa and HeLa/Neg-siRNA had similar cell cycle distributions, HeLa/ Neg-siRNA was used as the control cells in order to remove the effect of the plasmid vector (as before). It is known that on response to X-rays, a lower LET (linear energy transfer) radiation, mammalian cells normally are arrested $(\leq 10 \mathrm{~h})$ in G1, which is followed by DNA repair and/or apoptosis (21). At $4 \mathrm{~h}$ post-radiation in this study, the percentage in the G1 phase of $\mathrm{HeLa} / \mathrm{Ku} 80$-siRNA was $(55.45 \pm 3.49) \%$, which was lower compared to $(63.59 \pm 2.64) \%$ of the HeLa/Neg-siRNA $(\mathrm{P}<0.05)$. $\mathrm{S}$ arrest appeared in $\mathrm{HeLa} / \mathrm{Ku} 80-$ siRNA cells $(36.41 \pm 3.05) \%$ of the time compared to $(25.90 \pm 2.16) \%$ of the HeLa/Neg-siRNA cells $(\mathrm{P}<0.05)$. HeLa/Neg-siRNA or HeLa/Ku80-siRNA cells that were transiently transfected with DNA-PKcs-siRNA both showed decreases in G1 arrest and increases in S arrest, similar to changes seen in the HeLa/Ku80-siRNA cells (Fig. 3C).

$\mathrm{HeLa} / \mathrm{Ku} 80$-siRNA and HeLa/Neg-siRNA cell lines were both found to accumulate in the G2/M phase between 24 to $72 \mathrm{~h}$ after 6 Gy X-ray irradiation, and at $48 \mathrm{~h}$, the percentages were at highest levels for both, reaching above $80 \%$. The cells released from the accumulation of the $\mathrm{G} 2 / \mathrm{M}$ phase and reentering the cell cycle resulted in apoptosis at $72 \mathrm{~h}$. However, there were no obvious differences $(\mathrm{P}>0.05)$ in the cell proportions observed 

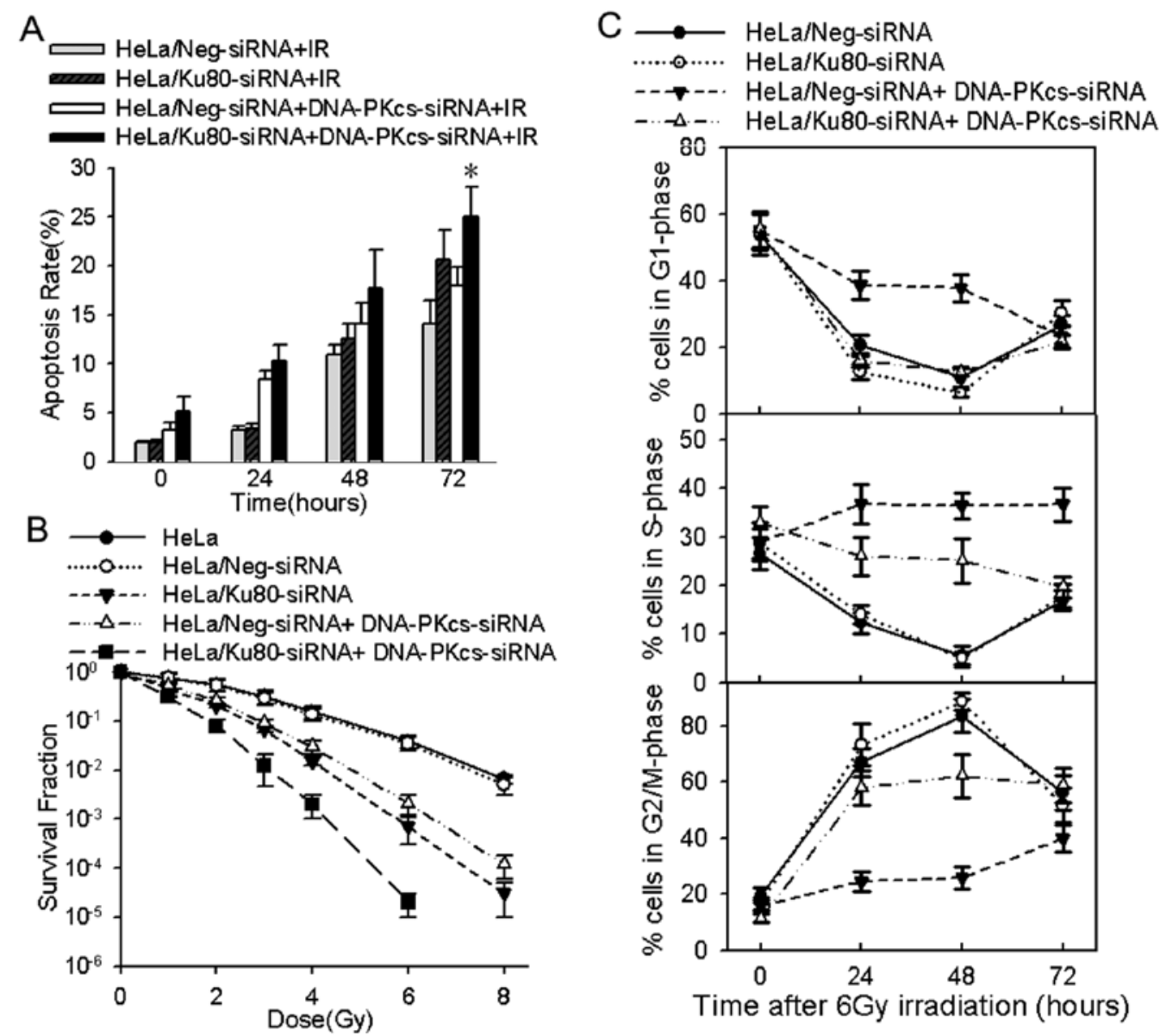

Figure 3. Radiobiology variability of HeLa after suppression of DNA-PKcs and Ku80 both singly and in combination with siRNA. (A) HeLa cells were transfected with Ku80-siRNA and/or DNA-PKcs-siRNA was irradiated for $6 \mathrm{~Gy}$ X-ray and was harvested at 24, 48 and $72 \mathrm{~h}$ after irradiation. Apoptosis rates (subG1) were analyzed by FACS. "P<0.05. (B) HeLa, HeLa/Neg-siRNA, HeLa/Ku80-siRNA, and cells cotransfected with DNA-PKcs-siRNA for 48 h were irradiated at the indicated dose and were assessed for clonogenic survival assay as described in 'Materials and methods'. (C) HeLa cells were transfected with Ku80-siRNA and/or DNA-PKcs-siRNA was irradiated with 6 Gy X-rays. All cells were harvested at 24,48 and $72 \mathrm{~h}$ after irradiation, and were analyzed by FACS as described in 'Materials and methods'. The cell percents in G1, S and G2/M phase are shown, respectively. All illustrated data are presented as mean based on three separate experiments.

Table I. Response of irradiated cell lines.

\begin{tabular}{lcccc}
\hline Cell lines and treatment & $\begin{array}{c}\text { Surviving fraction } \\
\text { at 2 Gy (SF2) }\end{array}$ & $\alpha$ & $\beta$ & $\begin{array}{c}\text { DRF of } 10 \% \\
\text { cell survival }\end{array}$ \\
\hline HeLa & $0.53 \pm 0.08$ & $0.19 \pm 0.02$ & $0.06 \pm 0.01$ & \\
HeLa/Neg-siRNA & $0.51 \pm 0.07$ & $0.19 \pm 0.02$ & $0.07 \pm 0.01$ & 1 \\
HeLa/Ku80-siRNA & $0.20 \pm 0.05$ & $0.70 \pm 0.03$ & $0.06 \pm 0.02$ & 1.68 \\
HeLa/Neg-siRNA + DNA-PKcs-siRNA & $0.25 \pm 0.03$ & $0.55 \pm 0.03$ & $0.07 \pm 0.02$ & 1.50 \\
HeLa/Ku80-siRNA + DNA-PKcs-siRNA & $0.08 \pm 0.01$ & $1.09 \pm 0.04$ & $0.09 \pm 0.02$ & 2.47 \\
HeLa/Neg-siRNA + LY294002 & $0.21 \pm 0.02$ & $0.63 \pm 0.04$ & $0.08 \pm 0.03$ & 1.63 \\
HeLa/Ku80-siRNA + LY294002 & $0.03 \pm 0.01$ & $1.51 \pm 0.06$ & $0.16 \pm 0.03$ & 3.36 \\
\hline
\end{tabular}

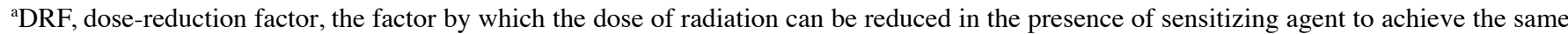
level of cell killing in the absence of the sensitizing agent, were calculated as the dose required to give $10 \%$ cell survival (90\% clonogenic cell killing) from fitted clonogenic survival curves for transfected or treated cells; in order to remove the effect of the plasmid vector, HeLa/NegsiRNA was regarded as control cells. Results are shown as mean values of each data point \pm standard error $(n=3)$.

at 24, 48 and $72 \mathrm{~h}$ after irradiation (Fig. 3C). HeLa/Ku80-siRNA transfected with DNA-PKcs-siRNA showed similar cell cycle trends as the $\mathrm{HeLa} / \mathrm{Ku} 80$-siRNA cells, accumulating at the
G2/M phase until $48 \mathrm{~h}$ post-irradiation, though the degree of change was not as pronounced (Fig 3C). However, HeLa/ Neg-siRNA transfected with DNA-PKcs-siRNA displayed an 

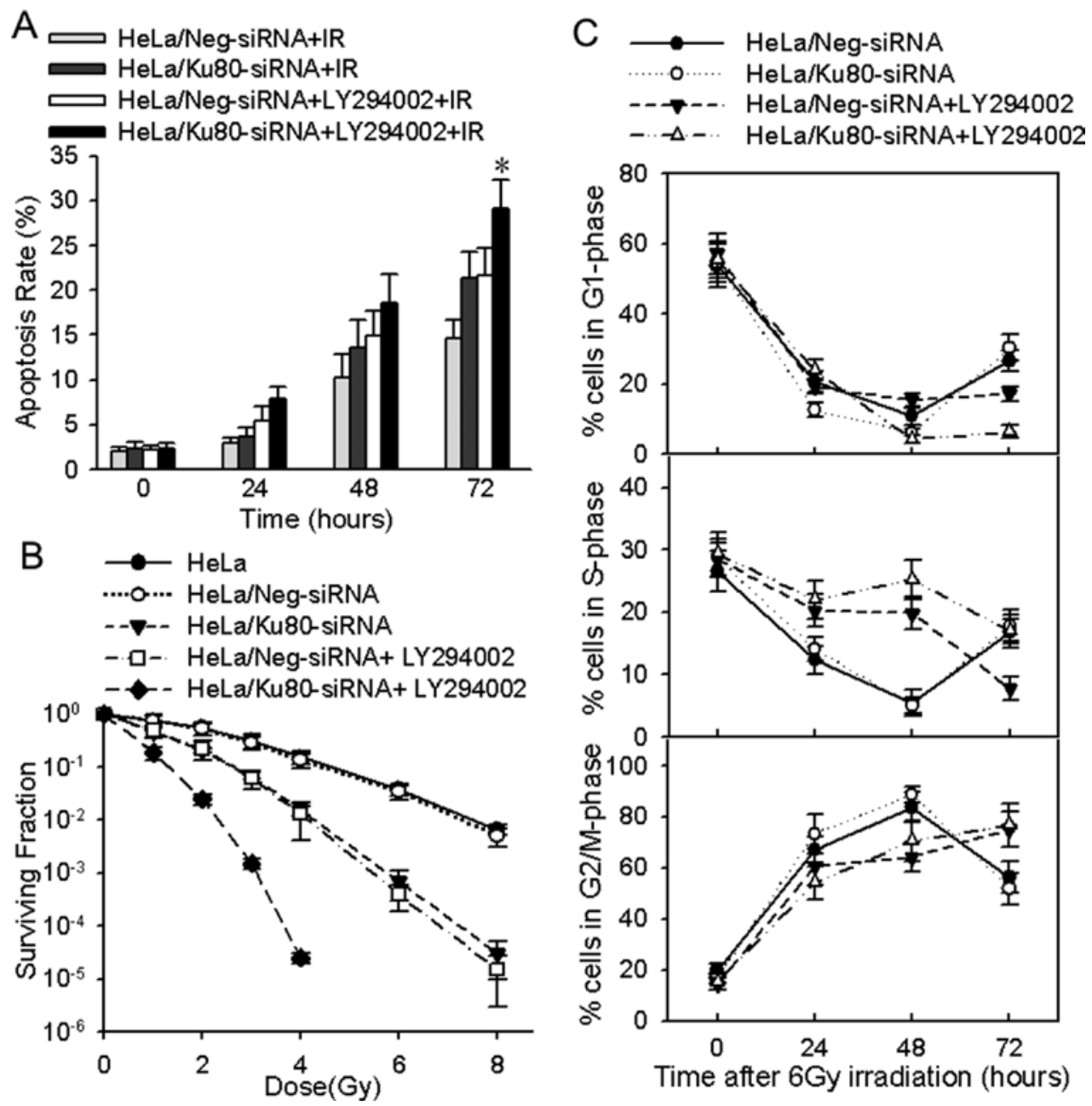

Figure 4. Radiobiology variability of HeLa after suppression of DNA-PKcs with LY294002 and/or Ku80 with siRNA both singly and in combination. (A) Cells pretreated with $50 \mu \mathrm{M}$ LY294002 for 30 min were irradiated for 6 Gy X-ray and were harvested at 24, 48 and $72 \mathrm{~h}$ after irradiation. Apoptosis rates (subG1) were analyzed by FACS. "P<0.05. (B) HeLa, HeLa/Neg-siRNA, and HeLa/Ku80-siRNA cells pretreated with $50 \mu \mathrm{M}$ LY294002 for 30 min were irradiated at the indicated dose and were assessed for clonogenic survival assay as described in 'Materials and methods'. (C) Cells pretreated with $50 \mu \mathrm{M}$ LY294002 for 30 min were irradiated with 6 Gy X-rays. All cells were harvested at 24, 48 and $72 \mathrm{~h}$ after irradiation, and were analyzed by FACS as described in 'Materials and methods'. The cell percents in G1, S, and G2/M phase are shown, respectively. All illustrated data are presented as mean based on three separate experiments.

interesting phenomenon; in contrast to the other cell lines, the cell cycle initially accumulated in the G1 phase for a long time, then entered the $\mathrm{S}$ phase and $\mathrm{G} 2 / \mathrm{M}$ phase slowly, in a step by step manner. The percentage of cells in the G2/M phase still did not get to a vertex even at $72 \mathrm{~h}$ post-irradiation. The percentage of cells in the G1 phase decreased gradually, though it did not reach the lowest point even at $72 \mathrm{~h}$ post-irradiation. In contrast, the other cell lines had reached the inflection point at $48 \mathrm{~h}$ post-irradiation, similar to $\mathrm{G} 2 / \mathrm{M}$ phase accumulation.

The effect of LY294002 on radiobiology of HeLa in the absence of Ku80. LY294002 inhibited the activity of two DSB repair proteins: DNA-PKcs and ATM. The apoptosis analysis displayed that the apoptotic rate of the HeLa cells treated with both Ku80-siRNA and LY294002 were higher than that of other cells ( $\mathrm{P}<0.05$, Fig. 4A). Even HeLa/Neg-siRNA showed increased radiosensitivity after pretreatment with LY294002, and the SF2 and DRF at $10 \%$ cell survival compared to the control cells were $0.21 \pm 0.02$ and 1.63 , respectively. HeLa in the absence of Ku80 and pretreated with LY294002 resulted in cells that were even more sensitive to X-rays than HeLa/ Ku80-siRNA transfected with DNA-PKcs-siRNA. The SF2 and DRF at $10 \%$ cell survival compared to the control cells were $0.03 \pm 0.01$ and 3.36, respectively (Fig. 4B and Table I).

The irradiation of LY294002-treated cells led to a different cell cycle distribution, with HeLa/Neg-siRNA and HeLa/ Ku80-siRNA showing similar accumulation in the G2/M phase (Fig. 4C). Similar to HeLa/Neg-siRNA transfected with DNA-PKcs-siRNA (Fig. 3C), the cells continued to slowly accumulate in the G2/M phase and did not reach the culminating point even at $72 \mathrm{~h}$ post-irradiation.

\section{Discussion}

It is known that the NHEJ pathway plays a crucial role in repairing DSBs during radiation in mammalian cells (4). In order to investigate the role of DNA-PK, reconstructed plasmids that could transcribe siRNA in cells were applied to inhibit the expression of Ku80. Human cervical adenocarcinoma cell line HeLa, also a mammalian cell line, was 
A

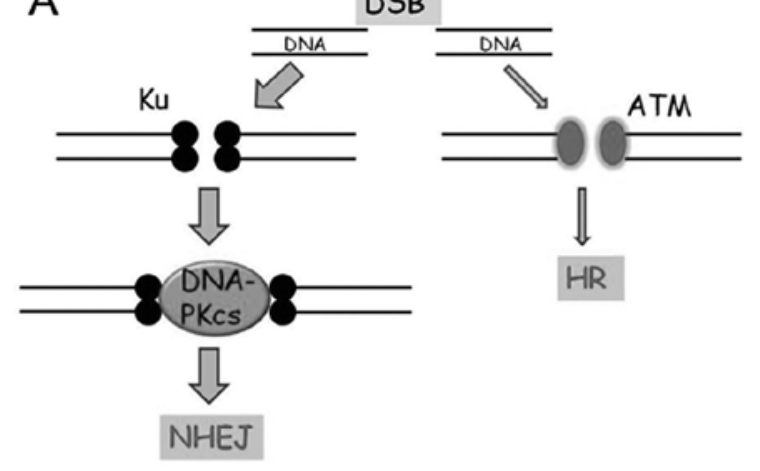

B
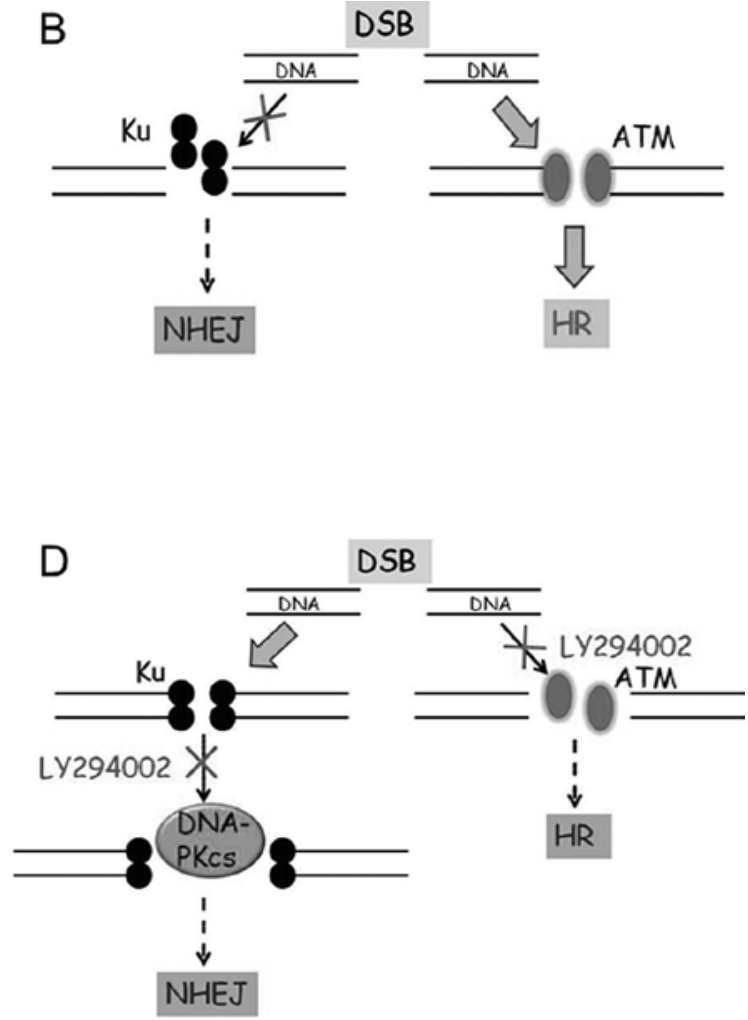

Figure 5. Schematic of two repair pathways of DSB. (A) Normal. (B) Ku80-defective. (C) DNA-PKcs-defective. (D) LY294002-treating cells. Ku means Ku heterodimer, including Ku70 and Ku80.

applied as a guide. We selected a stable transfection cell clone where $>96 \%$ of the Ku80 protein was silenced. While X-ray sensitivity of this clone was not obviously increased, the DRF was only 1.68 compared to the control cells. However, retransfection with siRNA targeting DNA-PKcs noticeably enhanced the radiosensitivity of $\mathrm{HeLa} / \mathrm{Ku} 80$-siRNA (DRF at $10 \%$ cell survival reached 2.47 , in comparison to the control cells). We know that Ku80 and DNA-PKcs are both components of DNA-PK and play same roles in the NHEJ pathway. Investigating if the two proteins play the same role upstream and downstream in the same pathway, the defecting of one protein and the knocking down of another nevertheless did not change the role of the pathway. This conclusion can be confirmed by the study that no effect was observed in M059J, which is a DNA-PKcs-deficient malignant glioma cell line after Ku80 antisense treatment (22). In order to explain the phenomenon that co-transfection with siRNAs targeting DNA-PKcs and Ku80 made the cells much more radiosensitive than transfection only with Ku80-targeting siRNA, we considered the following possible reasons. First, there are enormous differences in DSB repair protein expression between all kinds of eukaryotes, with human cells typically having more Ku80 and DNA-PKcs than rodents cells $(23,24)$, and the expressive differences are even displayed between different human cells $(25,26)$. Second, Ku80 proteins are abundant in human cells, so even a residual less than $4 \%$ of Ku 80 protein could act to rejoin many DSBs. Third, DNA-PKcs can also bind to, and is activated by, free DNA ends in the absence of $\mathrm{Ku}$ $(27,28)$. Fourth, there is competition or a sequence between the NHEJ and HR pathways (reviewed in refs. 3 and 11), with several studies suggesting that when NHEJ is impaired, HR seems to increase and vice versa (29-31). Other studies have raised the possibility that NHEJ is the initial pathway that is attempted; when it fails, HR might take over $(32,33)$.

Ionizing irradiation (IR) induces arrests in the G1, S and $\mathrm{G} 2 / \mathrm{M}$ phases of cell cycle in $<10 \mathrm{~h}$ (34); the work presented here demonstrated that the control cells were arrested in the G1 phase at $4 \mathrm{~h}$ after $6 \mathrm{~Gy}$ irradiation, just like other mammalian cells with wide-type p53. While HeLa cells inhibited Ku80 and DNA-PKcs both individually or in combination, they were arrested in the $S$ phase at $4 \mathrm{~h}$ after 6 Gy irradiation, confirmed results reported previously (35). Similar to reasons explained above, the DSB repair was compensated by the HR repair pathway when the NHEJ proteins were inhibited, so the cells were arrested in the $\mathrm{S}$ phase in order to allow the DSBs to be repaired by the HR protein that plays a main role in the late $\mathrm{S}$ and $\mathrm{G} 2 / \mathrm{M}$ phases $(6,7,35)$.

As reported by other investigators $(36,37)$, the increased number of G2/M cells that were detected several hours ( $\geq 24 \mathrm{~h}$ ) after irradiation in this study was likely due to an accumulation of cells that had been in earlier phases of the cell cycle because of radiation exposure. Our results suggested that regardless of HeLa cell transfection (only with negative-siRNA or with Ku80-siRNA), they all accumulated at the G2/M phase, while no obvious difference was discovered. But when we transfected siRNA targeting DNA-PKcs into the control HeLa cells and exposed them with 6 Gy X-ray irradiation, the result was different; the cell cycle initially accumulated in the G1 phase for a long time, then entered the $\mathrm{S}$ and $\mathrm{G} 2 / \mathrm{M}$ phases step by step at a rate far slower than the other cells. Interestingly, when 
the HeLa/Ku80-siRNA cells were transfected with the DNA-PKcs-siRNA and exposed to 6 Gy X-ray irradiation, the distribution of the cell cycle was like that of the HeLa/Ku80siRNA cells. All of the above results meet at a single conclusion, that the roles of Ku80 and DNA-PKcs in the cell cycle response are not the same, with DNA-PKcs perhaps acting in G2/M accumulation and being associated with Ku80, just like their roles in DSB repair. As shown in Fig. 5A, when DSB is induced, NHEJ proteins, Ku80 and Ku70, first bind to the free DNA end, and later recruit DNA-PKcs to complete the rest of the work $(3,5)$. When the Ku protein is defective (shown in Fig. 5B), the HR proteins RAD51, RAD52 and ATM can bind to the DNA end and HR will repair the DSB. If DNA-PKcs is defective instead of the Ku protein (shown in Fig. 5C), considering that the DNA ends were occupied by the Ku protein, the HR protein ATM can not bind to these ends and DSBs cannot be rejoined (31). We discovered a similar situation in the cell cycle response after X-ray irradiation. It is presumed that NHEJ and HR proteins both take part in the cell cycle response, with Ku80 acting first and later inducing the participation of the DNA-PKcs. When the Ku80 protein is inhibited, the HR protein can compensate for it completely because the role of these HR proteins in the cell cycle response was more powerful than that of the NHEJ proteins. As such, HeLa cell inhibition of Ku80 showed the same level of G2/M accumulation compared to the control cells. When DNA-PKcs was inhibited, regarding the 'occupying' of the Ku protein, HR proteins could not join or compensate normally, resulting in a delay in the cell cycle. In cells cotransfected with the both kinds of siRNA and receiving radiation (Ku80 protein having been silenced 96\%), the HR protein could compensate for major portion of the function, and the residual $4 \%$ of the Ku80 protein still 'occupied' a small part of the 'sites' that could be applied by neither the DNA- PKcs nor the HR protein. This maybe is the most possible explanation as to why the $\mathrm{G} 2 / \mathrm{M}$ accumulation of the cotrans-fected cell line was slightly lower than that of the control cells and HeLa/Ku80-siRNA.

LY294002, a chemically specific PI 3-kinase inhibitor capable of blocking the activity of both NHEJ protein DNA-PK and HR protein ATM (shown in Fig. 5D) (19), was used to treat the cells before $6 \mathrm{~Gy} \mathrm{X}$-ray irradiation. The cells silenced by Ku 80 were more sensitive to $\mathrm{X}$-rays than cells inhibited by DNA-PKcs-targeted siRNA, which supports our presumption that the HR protein ATM could compensate major portions of the DSB repair function even in the absence of Ku80. Moreover, even when cells had $96 \%$ the Ku 80 proteins knocked down by siRNA or when control cells had no Ku80 suppression, both scenarios displayed the same cell cycle distribution after LY294002 treatment and irradiation. Regarding the control cells, where previous results showed that irradiation of the LY294002-treated cells caused an increase in the length of G2/M arrest compared to cells exposed only to radiation (38), the inhibition of DNA-PKcs and ATM may block the recognition of DNA damage and prevent the recruitment of repair capacity in damaged sites. As for $\mathrm{HeLa} / \mathrm{Ku} 80$-siRNA, although the HR protein ATM may compensate for DSB repair function in the absence of Ku80 and though ATM maybe the main target of LY294002 (38), LY294002-treated cells displayed that large DNA damage could not be repaired, displaying the same prolonged $\mathrm{G} 2 / \mathrm{M}$ accumulation.
In conclusion, Ku80 and DNA-PKcs are both DSB repair proteins, though there still are many unknown mechanisms regarding their DSB repair function, cell cycle response and other radiobiology characteristics. In addition, the co-inhibition of both NHEJ and HR proteins would be a suitable strategy to enhance the radiosensibility of cancer cells.

\section{Acknowledgements}

We are grateful to Dr Xiaoyu Tian (Cancer Biology Research Center, Tongji Hospital, Tongji Medical College, Huazhong University of Science and Technology) for providing the constructed DNA-PKcs-targeted siRNA-encoding plasmid. This study was supported by grants from the National Natural Science Foundation of China (Nos.30801351; 30672426; 30901587) and Wu Jieping Medical Foundation (3206720.10013).

\section{References}

1. Hoeijmakers JH: Genome maintenance mechanisms for preventing cancer. Nature 411: 366-374, 2001.

2. van Gent DC, Hoeijmakers JH and Kanaar R: Chromosomal stability and the DNA double-stranded break connection. Nat Rev Genet 2: 196-206, 2001.

3. Lieber MR, Ma Y, Pannicke U and Schwarz K: Mechanism and regulation of human non-homologous DNA end-joining. Nat Rev Mol Cell Biol 4: 712-720, 2003.

4. Lieber MR: The mechanism of double-strand DNA break repair by the nonhomologous DNA end-joining pathway. Annu Rev Biochem 79: 181-211, 2010.

5. Collis SJ, DeWeese TL, Jeggo PA and Parker AR: The life and death of DNA-PK. Oncogene 24: 949-961, 2005.

6. Ira G, Pellicioli A, Balijja A, et al: DNA end resection, homologous recombination and DNA damage checkpoint activation require CDK1. Nature 431: 1011-1017, 2004

7. Morrison C, Sonoda E, Takao N, Shinohara A, Yamamoto K and Takeda S: The controlling role of ATM in homologous recombinational repair of DNA damage. EMBO J 19: 463-472, 2000

8. Hill R and Lee PW: The DNA-dependent protein kinase (DNAPK): More than just a case of making ends meet? Cell Cycle 9: 3460-3469, 2010

9. Hartlerode AJ and Scully R: Mechanisms of double-strand break repair in somatic mammalian cells. Biochem J 423: 157-168, 2009.

10. Pawelczak KS and Turchi JJ: Purification and characterization of exonuclease-free Artemis: Implications for DNA-PK-dependent processing of DNA termini in NHEJ-catalyzed DSB repair. DNA Repair 9: 670-677, 2010.

11. Mahaney BL, Meek K and Lees-Miller SP: Repair of ionizing radiation-induced DNA double-strand breaks by non-homologous end-joining. Biochem J 417: 639-650, 2009.

12. Dahm K: Role and regulation of human XRCC4-like factor/ cernunnos. J Cell Biochem 104: 1534-1540, 2008.

13. Gullo C, Au M, Feng G and Teoh G: The biology of Ku and its potential oncogenic role in cancer. Biochim Biophys Acta 1765: 223-234, 2006.

14. Salles B, Calsou P, Frit $\mathrm{P}$ and Muller C: The DNA repair complex DNA-PK, a pharmacological target in cancer chemotherapy and radiotherapy. Pathol Biol 54: 185-193, 2006.

15. Zhang Q, Williams ES, Askin KF, Peng Y, Bedford JS, Liber HL and Bailey SM: Suppression of DNA-PK by RNAi has different quantitative effects on telomere dysfunction and mutagenesis in human lymphoblasts treated with gamma rays or HZE particles. Radiat Res 164: 497-504, 2005.

16. Elbashir SM, Harborth J, Lendeckel W, Yalcin A, Weber K and Tuschl T: Duplexes of 21-nucleotide RNAs mediate RNA interference in cultured mammalian cells. Nature 411: 494-498, 2001.

17. Brummelkamp TR, Bernards R and Agami R: A system for stable expression of short interfering RNAs in mammalian cells. Science 296: 550-553, 2002.

18. Hartley KO, Gell D, Smith GC, et al: DNA-dependent protein kinase catalytic subunit: a relative of phosphatidylinositol 3-kinase and the ataxia telangiectasia gene product. Cell 82: 849-856, 1995. 
19. Vlahos CJ, Matter WF, Hui KY and Brown RF: A specific inhibitor of phosphatidylinositol 3-kinase, 2-(4-morpholinyl)-8-phenyl4H-1-benzopyran-4-one (LY294002). J Biol Chem 269: 5241-5248, 1994.

20. Fuhrman CB, Kilgore J, LaCoursiere YD, Lee CM, Milash BA Soisson AP and Zempolich KA: Radiosensitization of cervical cancer cells via double-strand DNA break repair inhibition. Gynecol Oncol 110: 93-98, 2008.

21. Fei P and El-Deiry WS: p53 and radiation responses. Oncogene 22: 5774-5783, 2003.

22. Belenkov AI, Paiement JP, Panasci LC, Monia BP and Chow TY: An antisense oligonucleotide targeted to human Ku86 messenger RNA sensitizes M059K malignant glioma cells to ionizing radiation, bleomycin, and etoposide but not DNA cross-linking agents. Cancer Res 62: 5888-5896, 2002.

23. Meulle A, Salles B, Daviaud D, Valet P and Muller C: Positive regulation of DNA double strand break repair activity during differentiation of long life span cells: the example of adipogenesis. PLoS One 3: E3345, 2008

24. Finnie NJ, Gottlieb TM, Blunt T, Jeggo PA and Jackson SP: DNA-dependent protein kinase activity is absent in xrs- 6 cells: implications for site-specific recombination and DNA doublestrand break repair. Proc Natl Acad Sci USA 92: 320-324, 1995.

25. Smith GCM and Jackson SP: The DNA-dependent protein kinase. Genes Dev 13: 916-934, 1999.

26. Koike M: Dimerization, translocation and localization of Ku70 and Ku80 proteins. J Radiat Res 43: 223-236, 2002.

27. West RB, Yaneva M and Lieber MR: Productive and nonproductive complexes of $\mathrm{Ku}$ and DNA-dependent protein kinase at DNA termini. Mol Cell Biol 18: 5908-5920, 1998.

28. Hammarsten O and Chu G: DNA-dependent protein kinase: DNA binding and activation in the absence of $\mathrm{Ku}$. Proc Nat Acad Sci USA 95: 525-530, 1998.

29 Pierce AJ, Hu P, Han M, Ellis N and Jasin M: Ku DNA endbinding protein modulates homologous repair of double-strand breaks in mammalian cells. Genes Dev 15: 3237-3242, 2001.
30. Allen C, Kurimasa A, Brenneman MA, Chen DJ and Nickoloff JA: DNA-dependent protein kinase suppresses double-strand breakinduced and spontaneous homologous recombination. Proc Natl Acad Sci USA 99: 3758-3763, 2002.

31. Allen C, Halbrook J and Nickoloff JA: Interactive competition between homologous recombination and non-homologous end joining. Mol Cancer Res 1: 913-920, 2003.

32. Frank-Vaillant $M$ and Marcand S: Transient stability of DNA ends allows nonhomologous end joining to precede homologous recombination. Mol Cell 10: 1189-1199, 2002.

33. Delacote F, Han M, Stamato TD, Jasin M and Lopez BS: An xrcc4 defect or Wortmannin stimulates homologous recombination specifically induced by double-strand breaks in mammalian cells. Nucleic Acids Res 30: 3454-3463, 2002.

34. Maity A, McKenna WG and Muschel RJ: The molecular basis for cell cycle delays following ionizing radiation: a review. Radiother Oncol 31: 1-13, 1994.

35. Zhou XY, Wang X, Wang H, Chen DJ, Li GC, Iliakis G and Wang Y: Ku affects the ATM-dependent S phase checkpoint following ionizing radiation. Oncogene 21: 6377-6381, 2002.

36. Xu B, Kim ST and Kastan MB: Two molecularly distinct G2/M checkpoints are induced by ionizing irradiation. Mol Cell Biol 22: 1049-1059, 2002

37. Holgersson A, Heiden T, Castro J, Edgren MR, Lewensohn R and Meijer AE: Different G2/M accumulation in M059J and M059K cells after exposure to DNA double-strand break-inducing agents. Int J Radiat Oncol Biol Phys 61: 915-921, 2005.

38. Rosenzweig KE, Youmell MB, Palayoor ST and Price BD: Radiosensitization of human tumor cells by the phosphatidylinositol3-kinase inhibitors wortmannin and LY294002 correlates with inhibition of DNA-dependent protein kinase and prolonged G2-M delay. Clin Cancer Res 3: 1149-1156, 1997. 